\title{
EDITORIAL
}

\section{The development of the Paediatric Virology Study Group: Ten years in the making}

\author{
IOANNIS N. MAMMAS ${ }^{1}$, MARIA THEODORIDOU ${ }^{2}$ and DEMETRIOS A. SPANDIDOS ${ }^{1}$ \\ ${ }^{1}$ Department of Clinical Virology, School of Medicine, University of Crete, \\ Heraklion 71003; ${ }^{2}$ 1st Department of Paediatrics, 'Aghia Sophia' Children's Hospital, \\ University of Athens School of Medicine, Athens 115 27, Greece
}

Received November 2, 2016; Accepted December 16, 2016

DOI: $10.3892 /$ etm.2016.3998

\begin{abstract}
Almost ten years ago, in the spring of 2007, at the Wirral University Teaching Hospital in Wirral (UK), cytomegalovirus (CMV)-positive twins were treated at the local Neonatal Intensive Care Unit (NICU) (1). The management of this viral infection in both neonates required the collaboration of an extended scientific network in Merseyside and Manchester, including several experienced consultants in Neonatology, Paediatrics and Microbiology. Based on the current literature at that time, there was no relevant protocol for CMV infection in premature twins, the ganciclovir doses and the recommended duration of the treatment were not stated and the follow-up frequency of clinical, laboratory and imaging assessment were not well defined (1). Thus, the necessity of the valuable input of a specialized consultant having clinical experience on Neonatology as well as on recent advances on Clinical Virology was highlighted by all the members of the team. Moreover, the importance of the clinical training on viral infections occurring in neonates and children became more than clear for all junior doctors and specialty trainees (STs), who were interested in pursuing a career in Paediatrics.

Inspired by these twins, a group of young STs and junior researchers tried to bring together Clinical Virology with Paediatrics and created the Paediatric Virology Study Group (PVSG). The fundamental aim of the PVSG was to provide an array of research and educational activities on viral infections occurring in neonates and children dedicated to continuing medical education. The PVSG was open to scientists interested in the field of Paediatric Virology, either as basic scientists, researchers and virologists or as medical students, STs, consultant paediatricians, Paediatric Infectious Diseases (PID) physicians and allied health professionals (2).
\end{abstract}

Correspondence to: Professor Demetrios A. Spandidos, Department of Clinical Virology, School of Medicine, University of Crete, Heraklion 71003, Greece

E-mail: spandidos@spandidos.gr

Key words: Paediatric Virology, Paediatrics, Neonatology, education
The recent hallmark in the legacy of the PVSG was the 'Workshop on Paediatric Virology' dedicated to promoting international scientific exchange and cooperation of both clinicians and researchers on Paediatric Virology. This workshop was organized for the first time on the 10th of October 2015 as an official session of the "20th World Congress on Advances in Oncology' and the '18th International Meeting on Molecular Medicine' $(3,4)$. The key lectureships focused on neonatal infections in premature neonates, polio vaccine anniversary and hepatitis B genotyping. During this meeting, a detailed proposal of Paediatric Virology as a separate subspecialty in Paediatrics was presented (4), stimulating in the following months a very interesting debate, in which several worldwide leading scientists participated (5). The '2nd Workshop on Paediatric Virology' took place on the 8th of October, 2016, focusing on HIV-1, hepatitis B and C in Africa, Zika virus in pregnancy and infancy, multimodality imaging of viral infections in children and Paediatric A\&E viral infections. The PVSG provided the key points of all presentations from both meetings published in the Experimental and Therapeutic Medicine. In a country under continuous financial crisis, Greece, our efforts insist with our will to inspire Peadiatrics, with our will to be inspired by children's hopes and smiles.

\section{References}

1. Mammas I, Breen L, Rackham O and Hughes A: Congenital cytomegalovirus (CMV) infection in twins born by a CMV-negative mother. Acta Paediatr 97: 317, 2008.

2. Mammas IN, Sourvinos G and Spandidos DA: The paediatric story of human papillomavirus (Review). Oncol Lett 8: 502-506, 2014.

3. Mammas IN, Greenough A, Theodoridou M, Kramvis A, Christaki I, Koutsaftiki C, Koutsaki M, Portaliou DM, Kostagianni G, Panagopoulou P, et al: Current views and advances on Paediatric Virology: An update for paediatric trainees. Exp Ther Med 11: 6-14, 2016.

4. Mammas IN, Greenough A, Theodoridou M and Spandidos DA: Paediatric Virology: A new paediatric subspecialty? A proposal at the Workshop on Paediatric Virology, Athens, October 10, 2015. Exp Ther Med 11: 3-5, 2016.

5. Mammas IN and Spandidos DA: The subspecialty of Paediatric Virology: A 'mosaic tile' in future Paediatrics. Exp Ther Med 12: 539-540, 2016. 